\title{
Análisis microbiológico de úlceras de presión en pacientes del Centro Nacional de Rehabilitación (CENARE)
}

\author{
Karla Villalobos-Camacho, ${ }^{1}$ Mercedes Hernández-Guerrero, ${ }^{2}$ Susana Arteaga-Acevedo, ${ }^{3}$ Federico Montero-Mejía, ${ }^{3}$ \\ Fernando García. ${ }^{4}$
}

\begin{abstract}
Justificación y objetivo: Las úlceras de presión son áreas localizadas de tejido necrótico que tienden a desarrollarse cuando los tejidos blandos son comprimidos entre una prominencia ósea y una superficie externa. La principal complicación de estas lesiones es que pueden constituirse en focos primarios de infecciones bacterianas. El objetivo del presente estudio fue realizar un análisis microbiológico de las úlceras de presión en pacientes de las unidades de Lesionados Medulares, Neurotrauma y Fisiatría General del Centro Nacional de Rehabilitación para determinar cuáles bacterias aerobias predominan en dichas lesiones y determinar el perfil de susceptibilidad a los antibióticos de los aislamientos bacterianos.
\end{abstract}

\begin{abstract}
Métodos: En este estudio se incluyeron 50 muestras recolectadas de 35 úlceras de presión en un total de 22 pacientes con daños en la médula espinal atendidos durante el período de agosto de 1998 a marzo de 1999. Las muestras fueron cultivadas en medios convencionales para el aislamiento de bacterias aerobias. La identificación de los aislamientos se realizó mediante pruebas bioquímicas y el sistema Vitek®. El perfil de susceptibilidad a antibióticos se realizó mediante la técnica Kirby-Bauer con respaldo por el sistema Vitek®.
\end{abstract}

Resultados: Los microorganismos más frecuentemente aislados fueron P. aeruginosa, Staphylococcus coagulasanegativa, S. intermedius y A. baumannii, los cuales presentaron resistencia contra diversos antibióticos. No se encontró ningún aislamiento de Staphylococcus aureus meticilina-resistente en las lesiones estudiadas. A lo largo del estudio se observó variabilidad en los perfiles de resistencia en aislamientos de P. aeruginosa de un paciente durante su internamiento, lo cual sugiere adquisición de genes de resistencia y sustitución de cepas. Los perfiles de resistencia sugieren una posible transmisión intrahospitalaria de cepas de P. aeruginosa, Staphylococcus coagulasa-negativa y $S$. intermedius.

Conclusiones: El presente estudio indica que la biota bacteriana encontrada en las úlceras de presión en pacientes con lesiones medulares del CENARE está constituida principalmente por P. aeruginosa, Staphylococcus coagulasa-negativa, $S$. intermedius y A. baumannii, que muestran resistencia a diversos antibióticos y que son probablemente de origen intrahospitalario.

Descriptores: úlceras de presión, resistencia a antibióticos, infecciones intrahospitalarias, Acinetobacter, Pseudomonas, Staphylococcus, Enterobacteriaceae.

Recibido: 4 de octubre de 2000.

Aceptado: 17 de abril de 2001.

\footnotetext{
Abreviaturas: CENARE, Centro Nacional de Rehabilitación; MRSA, Staphylococcus aureus meticilina-resistente; PSA, pruebas de susceptibilidad a antimicrobianos.

Laboratorio Clínico Milenium.

Laboratorio de Reactivos Químicos, Caja Costarricense de Seguro Social. Servicio de Fisiatría, Centro Nacional de Rehabilitación.

Centro de Investigación en Enfermedades Tropicales, Facultad de Microbiología, Universidad de Costa Rica.
}

Correspondencia: Fernando García, Centro de Investigación en Enfermedades Tropicales, Facultad de Microbiología, Universidad de Costa Rica, 2060 Ciudad Universitaria Rodrigo Facio, San José, Costa Rica. Fax (506) 225-2374. E-mail: fgarcia@cariari.ucr.ac.cr.
Las úlceras de presión son áreas localizadas de tejido necrótico que tienden a desarrollarse cuando los tejidos blandos son comprimidos entre una prominencia ósea y una superficie externa por un período prolongado de tiempo. ${ }^{1}$ Los sitios anatómicos más comunes para el desarrollo de las úlceras de presión incluyen el sacro, tuberosidades óseas, trocánter mayor, talones y maléolo lateral. Los cuatro factores involucrados con el desarrollo de estas úlceras son la presión, las fuerzas cortantes, la fricción y la humedad. ${ }^{1}$ Se han desarrollado varios sistemas para la clasificación de las úlceras de presión. El National Pressure Ulcer Advisory Panel (NPUAP) de los Estados Unidos ha clasificado las úlceras en cuatro grados. ${ }^{1}$ 
Las úlceras de grado I se caracterizan por ser un eritema de piel intacta, que puede convertirse en una abrasión confinada a la epidermis. Si se elimina la presión la úlcera de grado I eventualmente cicatrizará. Las úlceras de grado II se caracterizan porque penetran la epidermis, la dermis y la capa grasa subcutánea subyacente y cicatrizan si se elimina la presión y se protege mediante un apósito. Las úlceras de grado III usualmente están infectadas y se extienden a través de la grasa subcutánea al interior del músculo. Este tipo de úlcera cicatrizará por segunda intención, dejando una escara avascular. Las úlceras de grado IV se extienden hacia el hueso, volviéndose blandas y necróticas. Las complicaciones más importantes de las úlceras de presión son la infección, tanto por microorganismos aerobios como anaerobios, y otras originadas de la misma, incluyendo bacteremia, osteomielitis, tractos sinusoidales, endocarditis, meningitis, artritis séptica, amiloidosis, miasis y formación de pseudoaneurisma. ${ }^{1,2,3}$

El objetivo de este estudio fue realizar un análisis microbiológico de las úlceras de presión en pacientes que atienden el Centro Nacional de Rehabilitación (CENARE) para determinar cuáles bacterias aerobias predominan en dichas lesiones y su perfil de susceptibilidad a diversos antimicrobianos.

\section{Materiales y Métodos}

Pacientes y muestras: 22 pacientes, de 20 a 60 años de edad, de las Unidades de Lesionados Medulares, Neurotrauma y Fisiatría General del CENARE, quienes presentaban a su ingreso úlceras con grados de I a IV, fueron incluidos en este estudio realizado de agosto de 1998 a marzo de 1999. Las muestras de cada paciente se recolectaron a solicitud del médico tratante como parte del análisis rutinario y no se recolectaron muestras adicionales. El protocolo de estudio cumplió con los requerimientos del Comité de Etica y del Comité Científico del CENARE. A partir de 35 úlceras se recolectaron un total de 50 muestras incluyendo 1 de úlcera de grado I, 9 de úlceras de grado II, 17 de úlceras de grado III y 24 de úlceras de grado IV. El grado de las úlceras se determinó siguiendo los criterios del NPUAP. Las úlceras se encontraron más frecuentemente en la región sacra (37.1\%), la región isquiática (20.1\%), piernas (17.1\%), glúteos (14.3\%) y pies $(8.8 \%)$. Seis pacientes presentaron dos o más úlceras. A siete pacientes que permanecieron durante varios meses internados en el transcurso del estudio se les recolectaron muestras de las respectivas lesiones una vez al mes.

Procesamiento de las muestras: Las muestras de las úlceras se recolectaron por medio de raspados vigorosos del fondo de la lesión utilizando tres torundas de algodón estériles. ${ }^{4}$ Una primera torunda se utilizó para la inoculación de placas de agar sangre, agar MacConkey y agar manitol sal, la segunda para la preparación de un frotis y tinción de Gram y la tercera se inoculó en un tubo con caldo tioglicolato como cultivo de respaldo. Las placas de agar sangre fueron incubadas bajo condiciones capnofílicas por $18-24$ horas a $35^{\circ} \mathrm{C}$ y los otros medios de cultivo se incubaron en aerobiosis por 24 horas a $35^{\circ} \mathrm{C}$. Se procesaron aquellos aislamientos a partir de medios de cultivo con uno o dos morfotipos bacterianos predominantes y se descartaron medios de cultivo con tres o más morfotipos coloniales. La identificación de los aislamientos bacterianos se determinó mediante pruebas bioquímicas, ${ }^{5,6}$ mientras que las pruebas de susceptibilidad a los antimicrobianos (PSA) se realizaron mediante la técnica de difusión con disco. ${ }^{7}$ En algunos casos, la idenficación de las especies aisladas y las PSA se respaldaron en el sistema automatizado Vitek ${ }^{\circledR}$. Para las bacterias Gram-negativas se probaron amikacina, cefalotina, cefotaxime, ceftaxidima, ceftriaxone, ciprofloxacina, gentamicina, imipenem y tetraciclina, mientras que para las bacterias Gram-positivas se utilizaron amikacina, cefalotina, eritromicina, gentamicina, oxacilina, penicilina, tetraciclina y vancomicina.

Análisis estadístico: Se aplicó una prueba de hipótesis para la comparación de proporciones muestrales para el análisis de los resultados.

\section{Resultados}

De las 50 muestras de úlceras de presión analizadas se obtuvieron cultivos positivos en 45 , siendo 5 muestras negativas por bacterias aerobias. En 24 muestras (48\%) se obtuvieron cultivos con un solo microorganismo, mientras que en 21 muestras (42\%) se cultivaron dos o más microorganismos diferentes. Como se muestra en el Cuadro 1, se lograron obtener 68 aislamientos, siendo Pseudomonas aeruginosa la que presentó mayor porcentaje de aislamiento. Otras bacterias aisladas fueron, en orden decreciente, Staphylococcus coagulasa-negativa, S. intermedius y Acinetobacter baumannii.

Los aislamientos de Staphylococcus coagulasa-negativa no fueron identificados a nivel de especie. Adicionalmente se encontraron otras especies de la familia Enterobacteriaceae incluyendo Escherichia coli, Klebsiella y Enterobacter, mientras que Staphylococcus aureus fue aislado solamente en el $6 \%$ de las muestras analizadas. Al comparar los microorga-

\begin{tabular}{|c|c|c|}
\hline $\begin{array}{r}\text { Cuadro } \\
\text { Frecuencia de aislami } \\
\text { tes microorganismos a } \\
\text { de úlceras de presión (n } \\
22 \text { pacien } \\
\end{array}$ & $\begin{array}{l}1 \\
\text { iento de difer } \\
\text { partir de las } \\
=50 \text { ) obtenic } \\
\text { ites }\end{array}$ & $\begin{array}{l}\text { ren- } \\
\text { muestras } \\
\text { das de }\end{array}$ \\
\hline Microorganismo & $\begin{array}{l}\text { Número de } \\
\text { aislamientos }\end{array}$ & $\begin{array}{c}\% \text { de } \\
\text { aislamiento }\end{array}$ \\
\hline Pseudomonas aeruginosa & 21 & 42 \\
\hline Staphylococcus coagulasa-negativa & 17 & 34 \\
\hline Staphylococcus intermedius & 8 & 16 \\
\hline Acinetobacter baumannii & 7 & 14 \\
\hline Klebsiella ozaenae & 4 & 8 \\
\hline Staphylococus aureus & 3 & 6 \\
\hline Proteus mirabilis & 3 & 6 \\
\hline Klebsiella oxytoca & 2 & 4 \\
\hline Escherichia coli & 2 & 4 \\
\hline Enterobacter spp. & 1 & 2 \\
\hline
\end{tabular}


nismos aislados según el grado de úlcera, se observó que la biota bacteriana es similar para las úlceras de grado III y IV (Figura 1), pero las especies de la familia Enterobacteriaceae fueron más frecuentemente aisladas de las úlceras de grado IV $(25 \%)$ que de las úlceras de grado III $(13.8 \%)(\mathrm{p}<0.05)$. Además no se aisló $S$. aureus de las úlceras de grado IV.

Se obtuvo un aislamiento de Staphylococcus coagulasa-negativa de la única muestra recolectada a partir de una úlcera de grado I, mientras que a partir de las úlceras de grado II solamente se aislaron A. baumannii y Staphylococcus coagulasanegativa en asociación. De los 21 aislamientos obtenidos de P. aeruginosa, 16 de ellos se aislaron en asociación con otras bacterias, tanto con diversas especies de Staphylococcus como con especies de la familia Enterobacteriaceae, o con ambas (Cuadro 2). Los tres aislamientos de S. aureus recuperados en este estudio se obtuvieron en asociación con $P$. aeruginosa a partir de úlceras de grado III.

Los resultados de las PSA se muestran en los Cuadros 3 y 4. Para $P$. aeruginosa se observaron porcentajes altos de resistencia contra amikacina, cefotaxima, ceftazidima, ciprofloxacina y tetraciclina. En los aislamientos de A. baumannii se observaron también porcentajes altos de resistencia contra todos los antibióticos ensayados, con excepción de imipenen. Para K. ozaenae se observó resistencia total contra amikacina y ceftazidima y el $50 \%$ de los aislamientos mostraron resistencia contra ciprofloxacina y tetraciclina, mientras que contra ceftriaxona, gentamicina e imipenen se observó un 100\% de sensibilidad. En los aislamientos de Staphylococcus coagulasa-negativa se observaron altos porcentajes de resistencia contra la mayoría de los antibióticos ensayados. De estos aislamientos, 16 fueron resistentes a la oxacilina y a la eritromicina y 15 a la penicilina y gentamicina. Para las cepas de S. intermedius se evidenció un $87.5 \%$ de resistencia contra la eritromicina y la penicilina y un $62.5 \%$ de resistencia contra la oxacilina. Los tres aislamientos de $S$. aureus fueron resistentes a la penicilina, dos de los cuales mostraron también resistencia contra la eritromicina, mientras que todos fueron sensibles a los restantes antibióticos ensayados. Ninguno de los aislamientos de Staphylococcus mostró resistencia contra la vancomicina.

Los patrones de susceptibilidad fueron utilizados para la diferenciación de los aislamientos de una misma especie bacteriana. En dos pacientes se aisló $P$. aeruginosa en forma repetitiva en diferentes fechas a lo largo del estudio. En uno de ellos se aisló $P$. aeruginosa a partir de úlceras ubicadas en diversos sitios anatómicos en tres diferentes fechas. Durante su hospitalización este paciente recibió tratamiento con amikacina, cefalexina, cefalotina, ceftaxidime y ciprofloxacina para el control de las infecciones de sus úlceras de presión. Tres de los cuatro aislamientos de la primera fecha mostraron patrones de susceptibilidad muy similares entre sí y distintos del cuarto aislamiento. Los tres aislamientos de la segunda fecha mostraron patrones muy similares entre sí y se diferencian de los de la primera fecha en la resistencia contra

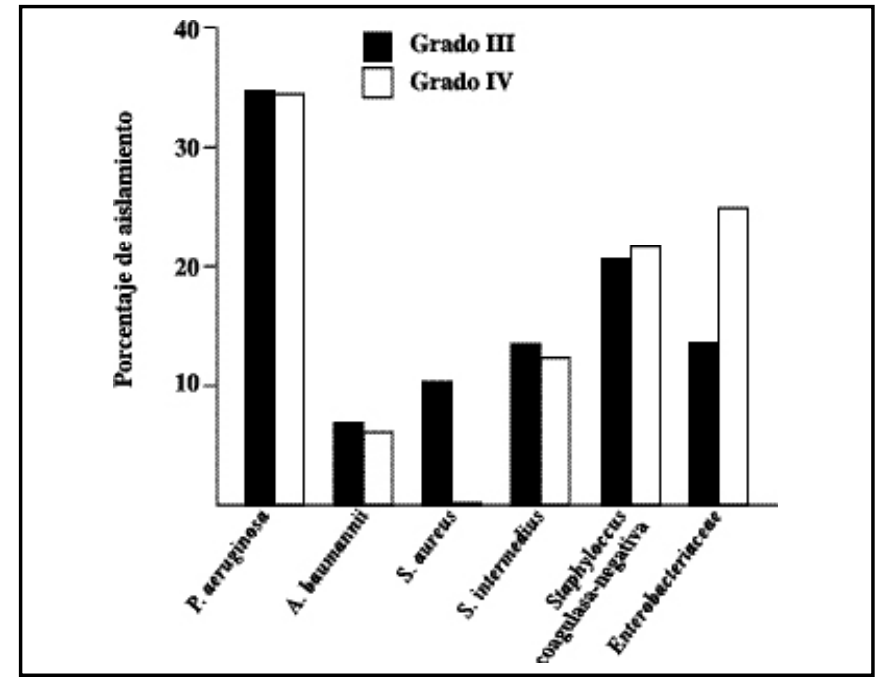

Figura 1. Microorganismos aerobios aislados de las úlceras de grado III $(n=29)$ y IV $(n=32)$ en pacientes con lesiones medulares. La diferencia observada en Enterobacteriaceae es estadísticamente significativa $(\mathrm{p}<0.05)$.

\begin{tabular}{|c|c|}
\hline \multicolumn{2}{|c|}{$\begin{array}{c}\text { Cuadro } 2 \\
\text { Asociaciones bacterianas en las muestras de las } \\
\text { úlceras de presión analizadas }\end{array}$} \\
\hline Asociaciones bacterianas & $\begin{array}{r}\text { Número de } \\
\text { Lestras }(n=50)\end{array}$ \\
\hline \multicolumn{2}{|l|}{$P$. aeruginosa con } \\
\hline Staphylococcus coagulasa-negativa & 6 \\
\hline S. aureus & 3 \\
\hline K. ozaenae & 2 \\
\hline S. intermedius & 1 \\
\hline Staphylococcus coagulasa-negativa y K. oxytoca & 1 \\
\hline S. intermedius y K. ozaenae & 1 \\
\hline K. oxytoca & 1 \\
\hline P. mirabilis & 1 \\
\hline A. baumannii con Staphylococcus coagulasa-negativa & 3 \\
\hline P. mirabilis con Enterobacter spp & 1 \\
\hline E. coli con Staphylococcus coagulasa-negativa & 1 \\
\hline Aislamiento de solo un microorganismo & 24 \\
\hline Cultivos negativos & 5 \\
\hline
\end{tabular}

ceftriaxona, ceftaxidima y cefotaxima. Los dos aislamientos de la tercera fecha mostraron patrones de susceptibilidad diferentes entre sí. El aislamiento del isquión derecho presentó el mismo patrón de resistencia que los aislamientos de la región sacra y del glúteo izquierdo de la primera fecha. Estos resultados indican, primero, la presencia de diferentes cepas de $P$. aeruginosa en diferentes sitios anatómicos, segundo, la posible adquisición de resistencia en el transcurso de la persistencia crónica de la bacteria y, tercero, la sustitución de cepas por otras con patrones de susceptibilidad diferentes.

Los patrones de susceptibilidad de los diferentes aislamientos de una misma especie fueron comparados entre sí con el fin de trazar posibles rutas de transmisión intrahospitalaria. 
Aislamientos de P. aeruginosa, Staphylococcus coagulasanegativa y $S$. intermedius obtenidos de pacientes que se encontraban en diferentes salones de la institución mostraron patrones de susceptibilidad prácticamente idénticos, sugiriendo una transmisión intrahospitalaria.

\section{Discusión}

La infección por diferentes tipos de microorganismos es la complicación más frecuentemente asociada a las úlceras de presión. La colonización representa el estado inicial del proceso infeccioso. En este estudio se realizó un análisis microbiológico para determinar la biota bacteriana aerobia presente en las úlceras de presión en pacientes del CENARE. $P$. aeruginosa y especies de Staphylococcus coagulasa-negativa fueron las bacterias más frecuentemente aisladas de las lesiones. Otras especies encontradas fueron A. baumannii, S. $\quad$ intermedius, S. aureus y de la familia Enterobacteriaceae. Aunque en la mayoría de las muestras (48\%) se logró aislar una única bacteria, en $42 \%$ de las muestras analizadas se encontraron dos o tres bacterias diferentes. Es interesante

\begin{tabular}{|c|c|c|c|c|c|c|}
\hline \multicolumn{7}{|c|}{$\begin{array}{c}\text { Cuadro } 3 \\
\text { Patrones de resistencia de los aislamientos de } \\
P . \text { aeruginosa, A. baumannii y } K . \text { ozaenae }\end{array}$} \\
\hline \multirow{2}{*}{ Antimicrobianos } & \multicolumn{2}{|c|}{ P. aeruginosa } & \multicolumn{2}{|c|}{ A. baumannii } & \multicolumn{2}{|c|}{ K. ozaenae } \\
\hline & $n=21$ & $\%$ & $\mathrm{n}=7$ & $\%$ & $\mathrm{n}=4$ & $\%$ \\
\hline Amikacina & 11 & 52.4 & 7 & 100.0 & 4 & 100.0 \\
\hline Cefotaxima & 10 & 47.6 & 7 & 100.0 & ND & ND \\
\hline Ceftazidima & 12 & 57.1 & 7 & 100.0 & 4 & 100.0 \\
\hline Ceftriaxona & 4 & 19.0 & 4 & 57.1 & 0 & 0 \\
\hline Ciprofloxacina & 13 & 61.9 & 7 & 100.0 & 2 & 50.0 \\
\hline Gentamicina & 7 & 33.3 & 7 & 100.0 & 0 & 0 \\
\hline Imipenem & 3 & 14.3 & 2 & 28.6 & 0 & 0 \\
\hline Tetraciclina & 13 & 61.9 & 7 & 100.0 & 2 & 50.0 \\
\hline
\end{tabular}

\begin{tabular}{|c|c|c|c|c|c|c|}
\hline \multicolumn{7}{|c|}{$\begin{array}{c}\text { Cuadro } 4 \\
\text { Patrones de resistencia de los aislamientos } \\
\text { de Staphylococcus }\end{array}$} \\
\hline \multirow[t]{2}{*}{ Antimicrobianos } & \multicolumn{2}{|c|}{$\begin{array}{c}\text { Staphylococcus } \\
\text { coagulasa-negativa } \\
\end{array}$} & \multicolumn{2}{|c|}{ S. intermedius } & \multicolumn{2}{|c|}{ S. aureus } \\
\hline & $\mathrm{n}=17$ & $\%$ & $\mathrm{n}=8$ & $\%$ & $n=3$ & $\%$ \\
\hline Amikacina & 2 & 11.8 & 2 & 25.0 & 0 & 0 \\
\hline Cefalotina & 7 & 41.2 & ND & ND & 0 & 0 \\
\hline Eritromicina & 16 & 94.1 & 7 & 87.5 & 2 & 66.7 \\
\hline Gentamicina & 15 & 88.2 & 0 & 0 & 0 & 0 \\
\hline Oxacilina & 16 & 94.1 & 5 & 62.5 & 0 & 0 \\
\hline Penicilina & 15 & 88.2 & 7 & 87.5 & 3 & 100.0 \\
\hline Tetraciclina & 4 & 23.5 & 3 & 37.5 & 0 & 0 \\
\hline Vancomicina & 0 & 0 & 0 & 0 & 0 & 0 \\
\hline
\end{tabular}

destacar el hecho que se observaron algunas asociaciones de microorganismos, como la de P. aeruginosa con Staphylococcus coagulasa-negativa. Sin embargo, el significado clínico o epidemiológico de estas asociaciones bacterianas no se determinó y será objetivo de futuros estudios.

Todos las especies bacterianas encontradas en este estudio son reconocidas por constituir parte de la biota indígena (flora normal) del ser humano en algún sitio anatómico, sea a nivel de piel o a nivel del tracto intestinal. ${ }^{8-10}$ Esto indica que el origen de estas bacterias podría ser de carácter endógeno, de la propia flora del paciente, o bien, adquirido de otros pacientes o del personal médico-asistencial de la institución. En efecto, el hallazgo de aislamientos de una misma especie bacteriana con patrones de susceptibilidad semejantes en diferentes pacientes sugiere fuertemente su adquisición nosocomial y la diseminación de microorganismos multirresistentes por medio del personal del hospital. Por otra parte, se demostró una variación de las características de resistencia en aislamientos de $P$. aeruginosa hallados en un paciente, ya sea debido a la adquisición de nuevos mecanismos de resistencia o al recambio de cepas luego de un tratamiento inicialmente exitoso. La mayoría de los aislamientos presentan diversos grados de resistencia contra diferentes antibióticos, lo cual es frecuente en bacterias de origen intrahospitalario. Los aislamientos de $P$. aeruginosa mostraron un porcentaje alto de resistencia contra aminoglicósidos, $\beta$-lactámicos y ciprofloxacina, mientras que los de A. baumannii encontrados en las muestras analizadas mostraron resistencia total contra aminoglicósidos, cefotaxima, ceftazidima, ciprofloxacina y tetraciclina. Aunque fueron relativamente pocos los aislamientos de $P$. aeruginosa y A. baumannii que mostraron resistencia contra imipenem, su hallazgo es de gran importancia por ser éste una de las pocas alternativas terapéuticas para el tratamiento de las infecciones por cepas multirresistentes de estas dos especies. La resistencia a imipenen en esas dos especies se debe a la producción de $\beta$-lactamasas con la capacidad de hidrolizar carbapenems. ${ }^{11,12}$ A A. baumannii se le considera actualmente como un agente emergente de infecciones nosocomiales, particularmente en las unidades de cuidados intensivos, donde el uso de antimicrobianos es intenso y el individuo presenta una mayor susceptibilidad a las infecciones. ${ }^{13}$ Así, la ocurrencia de $P$. aeruginosa y, principalmente, A. baumannii multirresistentes en las muestra analizadas debe ser tomada en consideración para el tratamiento empírico, de los pacientes con úlceras de presión en el CENARE, incluyendo dos o más antibióticos.

Aunque en el presente estudio no se encontró, $S$. aureus meticilina-resistente (MRSA) es también un importante agente de infecciones nosocomiales. ${ }^{14}$ Recientemente, la aparición de cepas de MRSA con sensibilidad disminuida a la vancomicina a planteado serios problemas en el tratamiento de estas infecciones nosocomiales. ${ }^{15}$ Afortunadamente, no se detectó ningún fenotipo de susceptibilidad disminuida a la vancomicina en los aislamientos de Staphylococcus aisladas. Sin embargo, otras especies de Staphylococcus meticilina resisten- 
te fueron encontrados con una frecuencia relativamente alta. Aunque a menudo las diversas especies de Staphylococcus coagulasa-negativa se consideran erróneamente como contaminantes, muchas especies del grupo, como S. epidermidis y $S$. haemolyticus, son ahora reconocidas como patógenos importantes, particularmente en ambientes intrahospitalarios. $^{16}$

La resistencia a antibióticos $\beta$-lactámicos en Staphylococcus se debe a la producción de enzimas con actividad $\beta$-lactamasa o de una proteína fijadora de penicillina denominada PBP2a (del inglés penicillin-binding proteins [PBP]) con actividad de serina-transferasa que muestra baja afinidad a antibióticos $\beta$-lactámicos. ${ }^{17,18}$ En la gran mayoría de los casos, la producción de PBP2a, codificada por el gen mecA, confiere resistencia prácticamente contra todos los antibióticos b-lactámicos, incluyendo aquellos que son resistentes a $\beta$-lactamasa, ${ }^{17}$ como meticilina y oxacilina. Recientemente se ha reportado un brote causado por una cepa de $S$. aureus expresando el inusual fenotipo penicilina-sensible/meticilina-resistente. ${ }^{19}$ Esta cepa posee el gen mecA pero no produce $\beta$-lactamasas. Un hallazgo interesante del presente estudio fue un aislamiento de Staphylococcus coagulasa-negativa mostrando también este fenotipo tan poco usual. Aunque las bases moleculares de este fenotipo no están aún elucidadas, es probable que sea el resultado de alguna mutación en mecA que halla aumentado su afinidad a la penicilina manteniendo una baja afinidad a la meticilina.

Los resultados del presente estudio indican, en conjunto, que la biota bacteriana aerobia de las úlceras de presión en pacientes del CENARE está constituida principalmente por microorganismos multirresistentes. Esto puede ser el resultado de la fuerte presión selectiva sobre las bacterias que constituyen la biota indígena por los tratamientos a los cuales están sometidos los pacientes con hospitalización reiterada o prolongada, lo cual favorece la adquisición horizontal de genes de resistencia. Alternativamente, algunos microorganismos pueden ser residentes intrahospitalarios que han adquirido los mecanismos de resistencia a lo largo del tiempo y colonizan el personal médico asistencial, el cual constituye la principal fuente de infección para los pacientes. Adicionalmente, los microorganismos multirresistentes causantes de infecciones de úlceras de presión y otras infecciones subsecuentes, representan un reto para el tratamiento de dichas lesiones y el bienestar de los pacientes.

\section{Agradecimientos}

Los autores agradecen al Dr. Gerardo Díaz Williams y a los revisores por sus sugerencias al manuscrito

\section{Abstract}

Background and aim: The pressure sores are areas of necrotic tissue that develop when soft tissues are compressed between a bone and an external surface. Bacterial infections are the main complication of the pressure sores. The aim of this study was to perform a microbiological analysis of pressure sores in order to determine the bacterial aerobic flora in patients admitted to the units of Medullary Injuries, Neurotrauma and General Fisiatry of the National Center of Rehabilitation and the antimicrobial susceptibility profiles of the isolates.

Methods: 50 samples collected from 35 pressure sores in 22 patients, during the period from August 1998 to March 1999 , were included in this study. Samples were cultured in conventional media for the isolation of aerobic bacteria. Identification of isolates were performed by standard biochemical tests and Vitek ${ }^{\circledR}$ system. Susceptibility tests were carried out by disk diffusion test and the Vitek® system.

Results: P. aeruginosa, coagulase-negative Staphylococcus, $S$. intermedius and $A$. baumannii were the most frequently isolated aerobic bacterial species, which showed resistance against several antibiotics. No methicillin-resistant Staphylococcus aureus was found. Several isolates of P. aeruginosa were isolated from different sores in a single patient. These isolates showed variability in the resistance patterns during the hospitalization, suggesting acquisition of resistance genes and reinfection with phenotypically distinguishable strains. The observed resistance patterns suggest a putative intrahospitalary transmission of $P$. aeruginosa, coagulase-negative Staphylococcus and S. intermedius.

Conclusions: Multiresistant $P$. aeruginosa, coagulase-negative Staphylococcus, S. intermedius and A. baumannii are the main bacterial components of the biota in the ulcer sores in patients of CENARE. Phenotypic traits suggest intrahospitalary dissemination of these bacteria among patients and institutional measures should be implemented to control such dissemination and to improve the use of antimicrobials against multiresistant bacteria.

\section{Referencias}

1. Kanj L, Wilking S, Phillips T. Continuing medical education: pressure sores. J Am Academ Derm 1998; 38: 517-541.

2. Patterson J, Bennett R. Prevention and treatment of pressure sores. J Am Geriatr Soc 1995; 43: 919-927.

3. Thomas D, Goode P, Tarquine P, Allman R. Hospital-acquired pressure ulcers and risk of death. J Am Geriatr Soc 1996; 44: 1435-1440.

4. Miller JM. A guide to specimen management in clinical microbiology. Washington, D.C.: American Society for Microbiology Press, 1996.

5. Shigei J. Test methods used in the identification of commonly isolated aerobic Gram-negative bacteria. En: Isenberg HD, ed. Clinical microbiology procedures handbook. Washington, D.C.: American Society for Microbiology Press, 1992; 1.19.1-1.19.111.

6. Pratt-Rippin K, Pezzlo M. Identification of commonly isolated aerobic Gram-positive bacteria. En: Isenberg HD, ed. Clinical microbiology procedures handbook. Washington, D.C.: American Society for Microbiology Press, 1992; 1.20.1-1.20.47.

7. National Committee for Clinical Laboratory Standards. Perfomance standards for antimicrobial disk susceptibility testing: approved standard M2-A4. Villanova, PA: National Committee for Clinical Laboratory Standards, 1997. 
8. Isenberg HD, D'Amato RF. Indigeneous and pathogenic microorganisms of humans. En: Murray PR, Baron EJ, Pfaller MA, Tenover FC, Yolken RH, ed. Manual of clinical microbiology, 6th edition. Washington, D.C.: American Society for Microbiology Press, 1995; 5-18.

9. Montgomerie JZ, Shick DG. pH and water content of Pseudomonas aeruginosa- and Klebsiella pneumoniae-colonized perineal skin of men with spinal cord injuries. J Clin Microbiol 1983; 18: 844-848.

10. Montgomerie JZ. Infections in patients with spinal cord injuries. Clin Infect Dis 1997; 25:1285-1292.

11. Bush K, Jacoby GA, Medeiros AA. A functional classification scheme for b-lactamases and its correlation with molecular structure. Antimicrob Agents Chemother, 1995; 39:1211-1233.

12. Da Silva GJ, Leitão R, Peixe L. Emergence of carbapenem-hydrolizing enzymes in Acinetobacter baumannii clinical isolates. J Clin Microbiol 1999; 37:2109-2110.

13. Marques MB, Brookings ES, Moser SA, Sonke PB, Waites KB. Comparative in vitro antimicrobial susceptibilities of nosocomial isolates of Acinetobacter baumannii and synergistic activities of nine antimicrobial combinations. Antimicrob Agents Chemother, 1997; 41:881-885.
14. Voss A, Doebbeling BN. The worldwide prevalence of methicillinresistant Staphylococcus aureus. Int J Antimicrob Agents, 1995; 5:101106.

15. Centers for Disease Control and Prevention. Interim guidelines for prevention and control of staphylococcal infections associated with reduced susceptibility to vancomycin. Morb Mort Weekly Rep, 1997; 46:626-635.

16. Kloos WE, Bannerman TL. Update on clinical significance of coagulase-negative staphylococci. Clin Microbiol Rev 1994; 7:117-140.

17. Chambers HF. Methicillin resistance in staphylococci: molecular and biochemical basis and clinical implications. Clin Microbiol Rev 1997; 10:781-791.

17. Massova I, Mobashery S. Kinship and diversification of bacterial penicillin-binding proteins and b-lactamases. Antimicrob Agents Chemother, 1998; 42:1-17.

18. Blanc DS, Petignat C, Moreillon P, Entenza JM, Eisenring MC, Kleiber $\mathrm{H}$, et al. Unusual spread of a penicillin-susceptible methicillin-resistant Staphylococcus aureus clone in a geographic area of low incidence. Clin Infect Dis 1999; 29:1512-1518. 